\title{
Evaluación in vitro de la resistencia a ciprofloxacina en biopelículas y poblaciones planctónicas de Pseudomonas aeruginosa de origen hospitalario
}

\author{
Christian Florián ${ }^{1}$, Débora Alvarado ${ }^{1}$, Juana Coha ${ }^{1}$
}

Resumen

Palabras clave

\begin{abstract}
Objetivo: Evaluar la susceptibilidad/resistencia in vitro de biopelículas y poblaciones planctónicas de Pseudomonas aeruginosa de origen hospitalario a la ciprofloxacina y compararlas con los resultados con las pruebas de susceptibilidad estándar obtenidos. Diseño: Estudio microbiológico. Lugar: Laboratorio de Microbiología Molecular, Facultad de Ciencias Biológicas, UNMSM. Material biológico: Cepas de Pseudomonas aeruginosa. Intervenciones: En cepas resistentes y sensibles obtenidas del Hospital del Niño, se realizó pruebas de concentración mínima inhibitoria (MIC); luego, se procedió a formar las biopelículas, en membranas de filtración y cultivos planctónicos en caldo, para ser sometidos ambos a diferentes concentraciones de ciprofloxacina y realizar el conteo en placas a intervalos de 40 minutos y a las 24 horas de exposición. Principales medidas de resultados: Susceptibilidad / resistencia in vitro de biopelículas y poblaciones planctónicas de Pseudomonas aeruginosa de origen hospitalario. Resultados: Al examinar la cepa sensible, ésta incrementa su resistencia en más de 200 veces, bajo la forma de biopelícula, encontrándose una diferencia poco significativa en las cepas resistentes, ya que la población comienza a disminuir alrededor de una concentración de $128 \mu \mathrm{g} / \mathrm{mL}$ de la droga. Conclusiones: Una misma cepa, como en el caso de la Pseudomona aeruginosa, tiene un comportamiento distinto a la ciprofloxacina, de acuerdo a su forma de crecimiento. Se recomienda la determinación del MIC en biopelículas de agentes etiológicos de infecciones recurrentes y crónicas que desarrollan el estado de biopelícula.
\end{abstract}

Pseudomonas aeruginosa; ciprofloxacina; agentes antiinfecciosos; infecciones por pseudomonas.
In vitro determination of ciprofloxacin resistance in Pseudomonas aeruginosa biofilms and hospital planktonic populations

\section{Abstract}

Objective: To assess in vitro sensitivity/resistance of biofilms and planktonic populations of Pseudomonas aeruginosa from a hospital environment to ciprofloxacin and to compare them against the results of standard susceptibility test obtained. Design: Microbiological study. Setting: Molecular Microbiology Laboratory, Faculty of Biological Sciences, UNMSM. Biologic material: Pseudomonas aeruginosa strains. Interventions: Resistant and susceptible strains obtained form Hospital del Niño were tested for minimum inhibitory concentration (MIC); then biofilms were formed on filtration membranes, and planktonic cultures in broth were tested with different ciprofloxacin concentrations, with plate count at 40 minutes intervals and 24 hours after exposure. Main outcome measures: In vitro susceptibility/resistance of hospital origin Pseudomonas

1 Laboratorio de Microbiología Molecular. Facultad de Ciencias Biológicas, UNMSM. Lima, Perú. aeruginosa biofilms and planktonic populations. Results: Sensitive strains increase their resistance more than 200 times under biofilm growth; no significant difference was found with resistant strains and both start to decrease their population at about $128 \mathrm{ig} / \mathrm{mL}$ drug concentration. Conclusions: The same strain, as in case of Pseudomona aeruginosa, has a differentresponse to ciprofloxacin, according to its growth. A MIC biofilm test of recurrent and chronic infections etiological agents is recommended.

Keywords: Pseudomonas aeruginosa; ciprofloxacin; anti-infective agents; pseudomonas infections.

\section{INTRODUCCIÓN}

Las biopelículas son comunidades complejas de microorganismos, presentes en ambientes naturales, que crecen sobre una superficie viva o inerte y que están encerradas en una matriz de exopolisacárido, formando asociaciones de una o múltiples 
especies con una organización semejante a la de los organismos multicelulares $\left({ }^{1}\right)$. Las biopelículas son muy difíciles de erradicar y son una fuente de infecciones persistentes. $\mathrm{Su}$ presencia en implantes médicos se debe al contacto de estos con fluidos corporales y a la reducción de la respuesta inmune en las proximidades de cuerpos extraños $\left({ }^{2,3}\right)$. En la biopelícula, las células bacterianas están distribuidas heterogéneamente formando microcolonias, donde solamente 10 a $20 \%$ del volumen total es celular; el resto es polisacárido, penetrado por minúsculos canales de agua. La tasa de crecimiento de la biopelícula es reducida, comparada con la de las bacterias planctónicas, en estado libre o de no asociación ${ }^{(4,5)}$.

Las infecciones causadas por biopelículas comparten algunas características clínicas. Se desarrollan preferentemente en superficies inertes o en tejido muerto y aparecen comúnmente en dispositivos médicos implantados; también, pueden formarse en tejido vivo, como en el caso de la endocarditis. Las biopelículas crecen lentamente, en uno o más lugares y este tipo de infecciones a menudo no producen una mejora en los síntomas. Las bacterias planctónicas secretan antígenos y estimulan la producción de anticuerpos, pero estos son poco efectivos para eliminar los microorganismos que conforman la película, incluso en personas con un excelente estado inmunológico $\left.{ }^{6}\right)$. La fibrosis quística, endocarditis, otitis media, periodontitis y prostatitis crónica parecen ser, todas, causadas por microorganismos asociados en biopelículas $\left({ }^{2}\right)$.

Una biopelícula es difícil de tratar con antimicrobianos, debido a que estos o son rápidamente inactivados o fallan en penetrar en su estructura, lo cual determina la resistencia de la biopelícula (biofilm) a estos compuestos; esta llega incluso a ser 1000 veces más alta. Estas mismas bacterias pueden tornarse sensibles cuando crecen bajo condiciones planctónicas $\left({ }^{7}\right)$. Se ha estudiado di- ferentes métodos para combatir películas bacterianas, incluyendo el uso combinado de varios agentes antimicrobianos, reportándose previamente combinaciones de tobramicina y piperacilina, tosufloxacina y eritromicina, clindamicina y claritromicina, por su efectividad en el tratamiento $\left({ }^{8}\right)$.

La Pseudomonas aeruginosa es una bacteria oportunista gram negativa, patógeno intrahospitalario en pacientes inmunodeficientes o con brechas en las defensas físicas del cuerpo, como las producidas por heridas, quemaduras o úlceras. Forma biopelículas en dispositivos médicos, siendo además agente causal de la fibrosis quística, cuyo tratamiento se asocia regularmente al desarrollo de resistencia a ciprofloxacina y a aminoglicósidos $\left({ }^{9}\right)$.

El incremento de la resistencia en biopelículas da como resultado la falla usual de los antibacterianos para su erradicación, aunque las pruebas de laboratorio demuestren sensibilidad a los antibióticos utilizados, por lo que se ha propuesto el desarrollo de biopelículas para usarlas en la determinación de la susceptibilidad a antibióticos $(7,10)$.

En el presente trabajo, se evalúa la susceptibilidad de cepas de $P$. aeruginosa seleccionadas de origen hospitalario, resistentes y sensibles a ciprofloxacina, bajo el estado de biopelícula y como cultivos planctónicos.

\section{MATERIALES Y MÉTODOS}

Se utilizó cepas de Pseudomonas aeruginosa, pertenecientes al cepario del Laboratorio de Microbiología Molecular, Facultad de Ciencias Biológicas, UNMSM $\left({ }^{11}\right)$, y al Hospital del Niño (cortesía del Dr. Rito Zerpa). Las cepas seleccionadas fueron nombradas PS1, PS2, PS3, PS4, PS5, PS6, PS7, PS8, PS9, PS10, PS11 y PS12 ${ }^{(11)}$ y PS13, PS14 (Hospital del Niño). 
La solución madre del antibiótico se preparó diluyendo la ciprofloxacina en agua destilada filtrada y se guardó a $-4{ }^{\circ} \mathrm{C}$. Las soluciones de uso fueron preparadas semanalmente, para asegurar el mantenimiento de la potencia del antibiótico.

La prueba de concentración mínima inhibitoria (MIC) se realizó en agar MüellerHinton con ciprofloxacina, conforme a las recomendaciones de Clinical and Laboratory Standards Institute para la prueba en placa (CLSI; NCCLS, 2004). Se sembró por diseminación $10 \mu \mathrm{L}$ de un cultivo reciente en caldo tripticasa soya (TSB) y se incubó a 37 ${ }^{\circ} \mathrm{C}$ por 24 horas. Las pruebas se realizó por duplicado y las concentraciones de antibiótico utilizadas fueron 0,$125 ; 0,25 ; 0,5 ; 1$; $2 ; 4 ; 8 ; 16 ;$ y $32 \mu \mathrm{g} / \mathrm{mL}$. Los resultados fueron registrados en razón al crecimiento o no de las cepas, frente a las diferentes concentraciones del antibiótico.

Para la preparación de biopelículas, se utilizó cultivos en caldo TSB, con opacidad según escala $N^{\circ} 1$ de Mc Farland. Se inoculó $5 \mu \mathrm{L}$ del caldo sobre membranas de nitrocelulosa, previamente esterilizadas con radiación UV por 20 minutos y colocadas sobre TSA. Se incubó a $37{ }^{\circ} \mathrm{C}$ por 48 horas, para asegurar la maduración de las biopelículas (Figura 1).

Para la evaluación de las cepas de $P$. aeruginosa en estado planctónico, se cultivó en TSB toda la noche, a $37{ }^{\circ} \mathrm{C}$, hasta alcanzar la escala $\mathrm{N}^{\circ} 1$ de Mc Farland. Se tomó una alícuota para su dilución en solución salina (SS) y se sembró en agar tripticasa soya (TSA), mediante la técnica de difusión, para obtener el número de células viables cultivables en el tiempo cero. Se añadió ciprofloxacina al cultivo restante, hasta alcanzar las concentraciones de $0,125,4$ y $16 \mu \mathrm{g} / \mathrm{mL}$. Se realizó una curva de crecimiento, tomando siete muestras cada 40 minutos y una final a las 24 horas de exposición al antibiótico; se mantuvo la incubación a $37{ }^{\circ} \mathrm{C}$. Se procedió con el re- cuento de igual manera que en el tiempo cero. Para el recuento y la expresión de resultados, se siguió las recomendaciones de la Food and Drug Administration (FDA) $\left({ }^{12}\right)$.

Las membranas maduras fueron colocadas sobre placas de TSA con ciprofloxacina. Solo una de las membranas (tiempo cero) fue colocada en SS y vortexada durante 1 a 2 minutos, para el desprendimiento de las células. Una muestra del homogenizado se diluyó en SS para el recuento en placa. Las demás membranas fueron sometidas al mismo tratamiento, cada 40 minutos; se tomó una muestra luego de $24 \mathrm{~h}$ de exposición al antibiótico. Las concentraciones de ciprofloxacina utilizadas fueron las mismas que para las células planctónicas. Para el recuento en placa sobre TSA, se sembró por diseminación hasta una dilución de $10^{-10}$; se incubó a $37{ }^{\circ} \mathrm{C}$ por 18 a 24 horas. Los resultados fueron expresados igual que para células viables cultivables planctónicas.

\section{RESULTADOS}

Las pruebas de concentración mínima inhibitoria (MIC) para ciprofloxacina dieron como resultado sensibilidad en 12 de las 14 cepas probadas; las cepas resistentes (PS13 y PS14) expresaron crecimiento hasta en $32 \mu \mathrm{g} / \mathrm{mL}$.

Para las pruebas de evaluación, se seleccionó las cepas PS9 (sensible), PS13 y PS14 (resistentes). Cabe resaltar que las cepas PS13 y PS14 mostraron un fenotipo diferente en agar Müeller-Hinton con antibiótico: PS13 como una colonia rugosa y PS14 como una colonia lisa y más plana. Se seleccionó al azar la cepa PS3 (sensible), para corroborar los resultados con la cepa PS9, datos no mostrados.

Las pruebas para la evaluación de las cepas se realizó con 3 concentraciones diferentes de antibiótico: baja $(0,125 \mu \mathrm{g} / \mathrm{mL})$, 
media $(4 \mu \mathrm{g} / \mathrm{mL})$ y alta $(16 \mu \mathrm{g} / \mathrm{mL})$, para ambos tipos de cultivo planctónico y biopelícula; las concentraciones fueron consideradas tomando en cuenta las reportadas en suero por la FDA y también los resultados de estudios previos $\left({ }^{13,14}\right)$. El desarrollo de las biopelículas en membrana se aprecia en la Figura 1. Las curvas de crecimiento con los valores promedio obtenidos para biopelículas y cultivos planctónicos a las concentraciones baja, media y alta respectivamente, se observa en la Figura 2.

La cepa sensible PS9 disminuyó su población visiblemente con una concentración baja de antibiótico en estado planctónico. Sin embargo, luego de 24 horas, aún cuando la población no desaparece, ésta disminuye en más de 5 unidades logarítmicas; a concentraciones media y alta la población planctónica, desciende a niveles no mensurables por la técnica utilizada para el recuento de microorganismos viables. La misma cepa en estado de biopelícula cambia su comportamiento frente a las mismas dosis de antibiótico; su población no disminuye en alguna de las concentraciones; incluso, luego de 24 horas la población parece mantenerse estable.

Las cepas resistentes PS13 y PS14 no mostraron variación alguna en su comportamiento, a pesar de exhibir fenotipos diferentes; resistieron en ambos tipos de cultivo de manera similar, a iguales concentraciones de antibiótico y no mostraron mayor variación en una comparación entre las mismas. En la Figura 3 se resume la reducción de la población en las 3 cepas, a diferentes concentraciones de ciprofloxacina, cuando son mantenidas en estado de biopelícula.

\section{DISCUSIÓN}

La mayoría de ensayos para poner en evidencia la susceptibilidad de una bacteria a un determinado antibiótico utiliza los métodos estandarizados de Kirby- Bauer o
MIC (en placa o en tubo) o ambos $\left({ }^{15-18}\right)$, los cuales son aplicados a bacterias que crecen en estado planctónico. Sin embargo, y a pesar de la conveniencia de estas pruebas debido a que nos dan resultados rápidos, no nos determinan la resistencia que se desarrolla en enfermedades en las cuales la bacteria forma biopelícula, como es el caso de Pseudomonas aeruginosa en otitis media, fibrosis quística o asociados a materiales en contacto con el cuerpo, como lentes de contacto y catéteres $\left(^{6}\right)$.

Los datos de evaluación de la sensibilidad muestran que, cuando se compara los valores MIC con los obtenidos en el enfrentamiento de la ciprofloxacina a biopelículas, se refleja una diferencia entre los estándares de la NCCLS (actualmente Clinical and Laboratory Standards Institute) probados con las cepas planctónicas y los aislados de Pseudomonas aeruginosa crecidos como biopelículas $\left({ }^{19}\right)$.

Estudios previos han demostrado que una terapia a base de ciprofloxacina, una quinolona con vida media de 3 a 15 horas y administrada cada 12 a 24 horas, brinda la ventaja de un buen rendimiento $\left({ }^{12,20}\right)$. La ciprofloxacina es una de las quinolonas to-

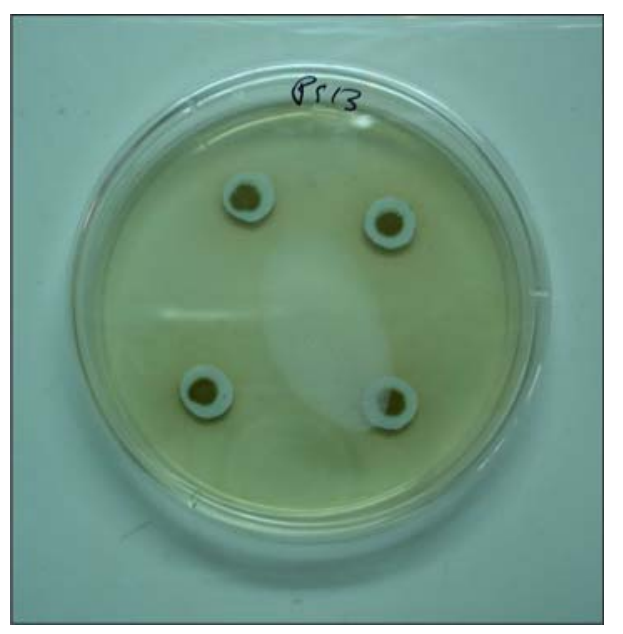

Figura 1. Crecimiento en placa de biopelículas de Pseudomonas aeruginosa sobre una membrana de filtración de nitrocelulosa (poro 0,22 $\mu \mathrm{m}$ de diámetro), luego de 48 horas de maduración. 


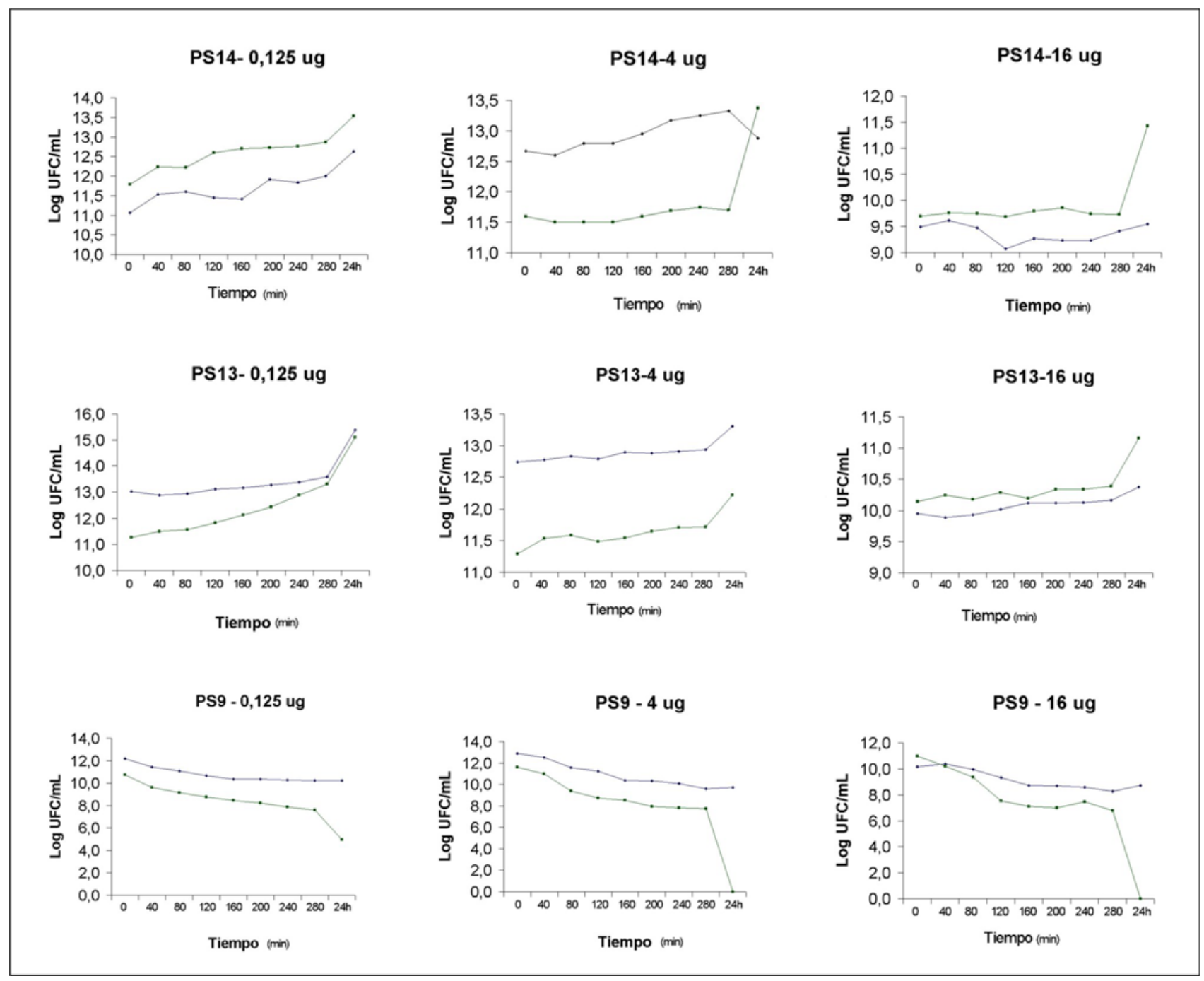

Figura 2. Curvas de crecimiento de biopelículas y cultivos planctónicos a 0,125, 4 y $16 \mu \mathrm{g} / \mathrm{mL}$ de ciprofloxacina, de las cepas PS14, PS13 y PS9 de $P$. aeruginosa. Los puntos representan la media de las mediciones realizadas; las líneas azules representan las biopelículas y las verdes los cultivos planctónicos.

davía recetada mayormente para el tratamiento de algunas infecciones en nuestro país, como las urinarias, fibrosis quística, pie diabético, otitis media y como alternativa a la tetraciclina en el tratamiento del cólera. Algunas de las quinolonas más recientes tienen un efecto tóxico para los pacientes y son usadas solo en algunos tipos de infecciones $\left({ }^{20}\right)$. No obstante, ciprofloxacina no contribuye de manera significativa en la erradicación de las infecciones, ya que una cepa sin resistencia que se encuentre formando una biopelícula incrementará su capacidad para mantenerse infecciosa debido a la protección que este estado le permite. Las pruebas que se realiza para la evaluación de sensibilidad de una bacteria como Pseudomonas aeruginosa, no están dando un resultado efectivo en caso de enfermedades infecciosas que son producidas por biopelículas, lo que se reflejaría en casos de infecciones persistentes y con poco o moderado efecto de los antibióticos suministrados. 


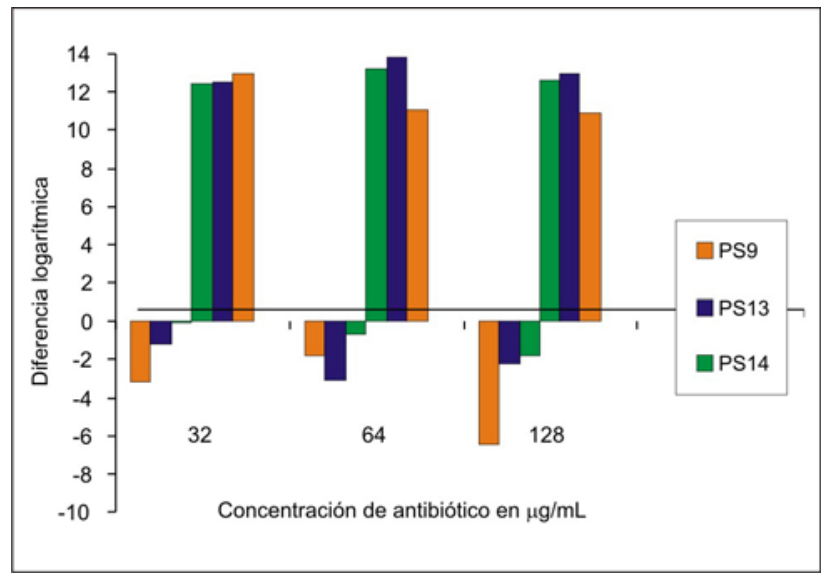

Figura 3. Disminución logarítmica de la población bacteriana de las cepas en estado de biopelícula a diferentes concentraciones de antibiótico. Los valores positivos representan la media al inicio y los valores negativos representan la disminución logarítmica después de 24 horas.

El estudio demuestra que existen diferencias para una misma cepa que, expresando susceptibilidad al MIC, es potencialmente resistente cuando adopta un estado de biopelícula. La biopelícula de la cepa PS9 sensible demostró una resistencia que superó en más de 200 veces $(32 \mu \mathrm{g} / \mathrm{mL})$ la concentración mínima inhibitoria a la que fue susceptible en estado planctónico; de la misma manera, PS3, la cepa que se usó para respaldar los resultados con PS9, mostró como biopelícula una disminución en la actividad de la ciprofloxacina, comparado con la prueba de sensibilidad estándar y con el cultivo planctónico en caldo.

Las infecciones de implantes médicos, como catéteres intravenosos, urinarios y peritoneales, son probablemente los mayormente estudiados $\left({ }^{2-4}\right)$. En estos estudios, los antibióticos suministrados en dosis muy altas han demostrado ser efectivos contra biopelículas; sin embargo, si muchos antibióticos tienen buena actividad en pruebas estándares de susceptibilidad, ésta no es la misma actividad contra biopelículas $\left({ }^{21}\right)$.

El uso de membranas, como soporte para el desarrollo de biopelículas en los ensayos de susceptibilidad a ciprofloxacina, resultó ser un método sencillo y barato que ha sido utilizado como modelo primitivo de algunas infecciones; este es mucho más fácil de utilizar y se puede hacer varias pruebas a la vez, además de requerir un menor espacio para las mismas $\left({ }^{22}\right)$.

Las biopelículas son consideradas generalmente resistentes a un amplio rango de agentes antimicrobianos, mostrando mayor tolerancia a antimicrobianos que las células planctónicas en fase logarítmica. Algunos antibióticos actúan con efectividad sobre células en crecimiento rápido; esto explicaría por qué la ciprofloxacina no pudo actuar sobre la cepa sensible PS9 a una concentración de $0,125 \mathrm{~g} / \mathrm{mL}$ en cultivo planctónico, debido probablemente a que la población alcanzó la fase estacionaria mucho antes de que el antibiótico pudiera actuar.

No obstante, en el caso de la biopelícula de la cepa sensible, se puede observar que la concentración de antibiótico requerido para su erradicación o para lograr su disminución a un nivel satisfactorio para un tratamiento, no se consigue sino hasta alcanzar niveles muy altos de la droga utilizada en este estudio. Aunque ésta, por lo general, no tiene efectos adversos, su uso en grandes dosis puede presentar problemas en pacientes, tales como trastornos hepáticos, renales y gastrointestinales reversibles $\left({ }^{12,20}\right)$.

Como se ve en la Figura 3, para la biopelícula de PS9 se alcanzó hasta 2,7 x $10^{4} \mathrm{UFC} / \mathrm{mL}$, luego de 24 horas, durante el ensayo con la dosis más alta de ciprofloxacina utilizada en la prueba (128 $\mu \mathrm{g} / \mathrm{mL}$ ). Probablemente, el hallazgo de una población reducida que significa el $0,00003 \%$ de la población inicial, consista en células llamadas 'persistentes' (persister cells), células no mutantes que tienen resistencia al antimicrobiano al cual es enfrentada la biopelícula y que se cree son producidas solo en ese estado $\left({ }^{23}\right)$. Este hallazgo es similar al encontrado por Spoering 
\& Lewis $\left({ }^{24}\right)$, en el cual la población de 'persistentes' de una biopelícula enfrentada a ácido peracético llegaba a ser $0,0001 \%$ de la población inicial. Sin embargo, este hecho fue descartado, porque no observó crecimiento en placa en una de las repeticiones y la resiembra de una colonia en caldo con antibiótico a $4 \mu \mathrm{g} / \mathrm{mL}$ no dio como resultado algún crecimiento.

La disminución significativa de valores poblacionales en las biopelículas resistentes puede deberse a la falta de un mayor número de repeticiones de la prueba, que hubieran reflejado cifras más reales. A pesar de este hecho, los valores correspondientes a la cepa sensible PS9 son similares a los esperados. No obstante, los cultivos de biopelículas de las cepas PS13 y PS14 resistentes mantienen su población alta, de manera similar a dosis menores. La disminución de la población es debida probablemente a factores físicos, como las concentraciones bajas de oxígeno en las capas más profundas de la biopelícula o la falta de nutrientes en algunas zonas de la misma $\left({ }^{8}\right)$. Incluso, cuando la reducción de la población de la biopelícula no ha sido completa, se distingue que las poblaciones tienen una significativa disminución, del orden de más de 2 a 3 unidades logarítmicas $\left({ }^{25,26}\right)$ : en las cepas sensibles, a partir de los $4 \mu \mathrm{g} / \mathrm{mL}$ y hasta los $64 \mu \mathrm{g} / \mathrm{mL}$ de ciprofloxacina (Figura 2) y en las cepas resistentes entre los $64 \mu \mathrm{g} / \mathrm{mL}$ y $\operatorname{los} 128 \mu \mathrm{g} / \mathrm{mL}$ (Figura 3).

Los datos obtenidos en las cepas PS13 (rugosa) y PS14 (lisa) no muestran una relación con la capacidad de resistencia en estado de biopelícula, sugiriendo que no hay mayor influencia del fenotipo. Tampoco se pudo apreciar relación alguna entre la fuerza de la adherencia notada en la facilidad o dificultad de desprendimiento de las biopelículas, tanto de parte de las cepas sensibles como de las cepas resistentes.

Los resultados indican que una misma cepa tiene un comportamiento distinto, de acuerdo a su forma de crecimiento, por lo que las pruebas de susceptibilidad tendrían que ser realizadas bajo estas dos formas, según sea el tipo de infección, sobretodo en infecciones crónicas o de difícil remisión, como es el caso de las causadas por Pseudomonas aeruginosa. Las futuras investigaciones deben apuntar hacia la estandarización de un método para obtener resultados comparables de los efectos de los antibióticos sobre biopelículas in vitro, para su aplicación como una prueba de rutina en pacientes donde se sospecha la participación de películas microbianas.

\section{REFERENCIAS BIBLIOGRÁFICAS}

1. Thurnheer T, Gmür R, Guggenheim B. Multiplex FISH analysis of a six-species bacterial biofilm. J Microbiol Methods. 2004;56:37-47.

2. Donlan R. Biofilms and device-associated infections. Emer Infect Dis. 2001;7(2):277-81.

3. Dunne M. Bacterial Adhesion: Seen any good biofilm lately? Clinical Microbiol Rev. 2002;15(2):155-66.

4. Costerton J, Lewandowski Z, DeBeer D, Caldwell D, Korber D, James G. Biofilms, the customized microniche. J. Bacteriol. 1994;176(8):2137-42.

5. Branda S, Vik $\AA$, Friedman L, Kolter R. Biofilms: the matrix revisited. Trends Microbiol. 2005;13(1):20-6.

6. Costerton J, Stewart P, Greenberg E. Bacterial biofilms: a common cause of persistent infections. Science. 1999;284:1318-22.

7. Høiby N. New antimicrobials in the management of cystic fibrosis. J Antimicrob Chemother. 2002;49:235-8.

8. Walters M, Roe F, Bugnicourt A, Franklin M, Stewart P. Contributions of antibiotic penetration, oxygen limitation, and low metabolic activity to tolerance of Pseudomnas aeruginosa biofilms to ciprofloxacin and tobramycin. Antimicrob Agents Chemother. 2003;47(1):317-23.

9. Wilson R, Dowling R. Pseudomonas aeruginosa and other related species. Thorax. 1998;53:213-9.

10. Anwar H, Dasgupta M, Costerton J. Testing the susceptibility of bacteria in biofilms to antibacterial agents. Antimicrob Agents Chemother. 1990;34(11):2043-6.

11. Coha J M, García R, Ramírez P, Delgado F, Sanchez N, Bazán A. Prevalencia de Pseudomonas aeruginosa de infantes quemados hospitalizados. Resúmenes Reunión científica del ICBAR-UNMSM, Abril 1992. Lima: UNMSM; 1992. p. 82.

12. FDA. (2004). Documento descriptivo sobre composición, farmacocinética, farmacología clínica, microbiología, dosis y uso de la ciprofloxacina [monografía en Internet]. Rockville, 
USA: FDA; 2004 [citado el 12 de noviembre de 2006]. Disponible en: http://www.fda.gov/medwatch/SAFETY/ 2004/jul PI/Cipro PI.pdf

13. Ashby MJ, Neale J, Knott S, Critchley I. Effect of antibiotics on non-growing cells and biofilms of Escherichia coli. J Antimicrob Chemother. 1994;33:443-52.

14. Ceri H, Olson M, Streamick C, Read R, Morck D, Buret A. The Calgary Biofilm Device: New technology for rapid determination of antibiotic susceptibilities of bacterial biofilms. J Clin Microbiol. 1999;37(6):1771-6.

15. Ibrahim-Elmagboul I, Livermore D. Sensitivity testing of ciprofloxacin for Pseudomonas aeruginosa. J Antimicrob Chemother. 1997;39:309-17.

16. Chumpitaz J, Medina J, Huamán A, Cigüeña S, Palomino S. Resistencia bacteriana en infecciones intrahospitalarias de vías urinarias. Rev Per Enf Infec Trop [revista en Internet]. 2001 [citado el 14 de noviembre de 2006];1(4). Disponible en. http://sisbib.unmsm.edu.pe/BVRevistas/SPEIT/ $2001 \mathrm{n} 4 /$ index.htm

17. Nuñez L, Soto A, Calmet E, Castillo M, Casalinda E. Evaluación clínica y de laboratorio de las infecciones producidas por Pseudomonas aeruginosa en el Hospital Arzobispo Loayza. Rev Per Enf Infec Trop [revista en Internet]. 2001 [citado el 14 de noviembre de 2006];1(4). Disponible en. http://sisbib.unmsm.edu.pe/BVRevistas/ SPEIT/2001 n4/index.htm

18. Horna G, Silva M, Vicente W, Tamariz J. Concentración mínima inhibitoria y concentración mínima bactericida de ciprofloxacina en bacterias uropatógenas aisladas en el Instituto Nacional de Enfermedades Neoplásicas. Rev Méd Hered. 2005;16(1):39-45.

19. NCCLS. Performance Standards for Antimicrobial Susceptibility Testing. Fourteenth Informational Supplement. 2004;24(1):M100-S14.
20. Solari L, Maguiña C. Nuevas y viejas quinolonas. Rev Med Hered. 2002;13(4):153-60.

21. Moskowitz S, Foster J, Emerson J,Burns J. Clinically feasible biofilm susceptibility assay for isolates of Pseudomonas aeruginosa from patients with cystic fibrosis. J Clin Microbiol. 2004;42(5):1915-22.

22. Anderl J, Franklin M, Stewart P. Role of antibiotic penetration limitation in Klebsiella pneumoniae biofilm resistance to ampicillin and ciprofloxacin. Antimicrob Agents Chemother. 2000;44(7):1818-24.

23. Brooun A, Liu S, Lewis K. A dose-response study of antibiotic resistance in Pseudomonas aeruginosa biofilms. Antimicrob Agents Chemother. 2000;44(3):640-6.

24. Spoering A, Lewis K. Biofilms and planktonic cells of $P$. aeruginosa have similar resistance to killing by antimicrobials. J Bacteriol. 2001;183(23):6746-51.

25. Cahen P, Lebourgeois M, Delacourt C. Serum bactericidal test as a prognostic indicator in acute pulmonary exacerbations of cystic fibrosis. Pediatrics. 1993;91(2):451-5.

26. Oldak E, Trafni E. Secretion of proteases by Pseudomonas aeruginosa biofilm exposed to ciprofloxacin. Antimicrob Agents Chemother. 2005;49(8):3281-8.

Manuscrito recibido el 12 de diciembre de 2006 y aceptado para publicación el 18 de diciembre de 2006.

Correspondencia:

Débora Alvarado

Laboratorio de Microbiología Molecular.

Facultad de Ciencias Biológicas, UNMSM.

Apdo. 720. Lima 1, Perú.

Correo-e: dalvaradoi@unmsm.edu.pe 